\title{
Metodología de participación ciudadana en el proceso de desarrollo rural endógeno en el caso Cantón Limón
}

\author{
Indanza.
}

\section{Methodology of citizen participation in the endogenous rural development process in the case of Cantón Limón Indanza}

\author{
Boris Adrián Argudo Domínguez. ${ }^{1}$ \& Yonimiler Castillo Ortega. ${ }^{2}$ \\ Recibido: 15-12-2020 / Revisado: 05-01-2021 /Aceptado: 12-01-202021/ Publicado: 05-02-2021
}

\begin{abstract}
.
DOI: https://doi.org/10.33262/concienciadigital.v4i1.1.1547

Introduction: Citizen participation in local planning activities can represent a great challenge for rural communities, due to factors such as territorial qualities, distances between communities, access to information and the use of technical language; however, participation is one of the most valuable instruments in strengthening democracies. Objective: To design a methodology that fosters greater openness to citizen participation in the endogenous rural development process of the Limón Indanza canton. Methodology: The study has a mixed approach, in which different instruments such as the survey, the interview and the focus group were applied to obtain information, while it ends with an intervention proposal through the participation methodology. Results: As main results it was found that citizen participation mechanisms are limited in the canton; there are contradictions between institutional and citizen perceptions regarding participation; and, citizens do not follow up on development projects proposed in the PDOT. Conclusion: A participatory methodology based on open government was proposed, through the LUDO model, with which the integration of organizations, social groups and actors of the Limón Indanza canton is sought.
\end{abstract}

\footnotetext{
${ }^{1}$ Universidad Católica de Cuenca, Posgrado, Maestría en Desarrollo Local Mención en Planificación y Ordenamiento Territorial, Cuenca, Ecuador, boris.argudo@gmail.com, ID de ORCID https://orcid.org/0000$0002-1412-7665$

${ }^{2}$ Universidad Católica de Cuenca, Posgrado, Maestría en Desarrollo Local Mención en Planificación

y Ordenamiento Territorial, Cuenca, Ecuador, ycastilloo@ucacue.edu.ec, ID de ORCID https://orcid.org/0000-0002-7710-5199
} 
Keywords: Endogenous Rural Development, Local Planning, Citizen Participation.

\section{Resumen.}

Introducción: La participación ciudadana en las actividades de planificación local puede representar un gran reto para las comunidades rurales, debido a factores como las cualidades territoriales, las distancias entre comunidades, el acceso a la información y el manejo del lenguaje técnico; no obstante, la participación es uno de los instrumentos más valiosos en el fortalecimiento de las democracias. Objetivo: Diseñar una metodología que propicie una mayor apertura a la participación ciudadana en el proceso desarrollo rural endógeno del cantón Limón Indanza. Metodología: El estudio es de enfoque mixto, en el cual se aplicaron diferentes instrumentos como la encuesta, la entrevista y el grupo focal para obtener información, en tanto que se finaliza con una propuesta de intervención mediante la metodología de participación. Resultados: Como resultados principales se encontró que los mecanismos de participación ciudadana son limitados en el cantón; existen contradicciones entre las percepciones institucional y ciudadana respecto a la participación; y, los ciudadanos no realizan el seguimiento a los proyectos de desarrollo planteados en el PDOT. Conclusión: Se propuso una metodología de participación basada en el gobierno abierto, a través del modelo LUDO, con el cual se busca la integración de organizaciones, grupos sociales y actores del cantón Limón Indanza.

Palabras claves: Desarrollo Rural Endógeno, Planificación Local, Participación Ciudadana.

\section{Introducción.}

El desarrollo económico local busca mejorar las condiciones de vida de una población específica, como un proceso de transformación orientado a superar los retos y dificultades, mediante determinadas acciones para el mejoramiento de las condiciones generales y el aprovechamiento más eficiente y sustentable de los recursos generando progreso en la localidad (Alburquerque \& Cortés, 2001).

Existe una conexión entre el desarrollo local y los distintos tipos de enfoques: regional, territorial, endógeno y descentralizado, cuyo objetivo común es la generación de desarrollo desde lo interno, que buscan entre sí potenciar las acciones locales mediante la capacitación y formación profesional del capital humano. Con la finalidad de impulsar el bienestar de las comunidades y de esta manera construir sociedades sustentables, los gobiernos democráticos, que se caracterizan por impulsar la participación ciudadana dentro de los espacios de planificación, han instaurado procesos de descentralización que fomentan el desarrollo endógeno.

El proceso de participación ciudadana, holístico, sinérgico y, a su vez, tan utópico, establece una relación horizontal gobierno-ciudadano, buscando siempre el predominio de una 
comunicación sana, constructiva y fluida que genere un ambiente armónico y equilibrado, a través de normas que regulen la interacción social; además, este proceso parte de la necesitad de identificar y analizar los problemas y soluciones a través de cinco etapas: diagnóstico, formulación, programación, ejecución y monitoreo y evaluación de los resultados, para administrar con acciones y recursos los objetivos establecidos por la institucionalidad y controlado por la ciudadanía (SENPLADES, 2012).

Para avanzar en un desarrollo rural sustentable y competitivo, algunos países de la región han creado instituciones y puesto en marcha programas que buscan activar procesos productivos, sociales y económicos. Hoy, en América Latina el desarrollo rural y, particularmente, la agricultura familiar, deben enfrentar una situación de globalización en la que resulta muy difícil ser competitiva. Para conocer mejor esta situación y plantear posibles caminos de solución, diversos organismos internacionales examinan la situación rural y cuentan con importante información para proponer políticas y diseñar estrategias sectoriales e institucionales.

En Ecuador, la exclusión del sector rural se ha transformado en una práctica municipal; por ello, es necesario comprender que en las áreas alejadas y zonas rurales están los pilares fundamentales de la economía nacional: áreas agroexportadoras (bananeras, cacaoteras, etc.), complejo hidrocarburífero (pozos petroleros, oleoductos, etc.) y las zonas ganaderas, las cuales deben ser aprovechadas e impulsadas en beneficio de las comunidades y de la nación.

Bajo este marco, la presente investigación tiene por objetivo diseñar una metodología que propicie una mayor apertura a la participación ciudadana en el proceso desarrollo rural endógeno en el cantón. Para cumplir con el objetivo general fue necesario lo siguiente: identificar las principales teorías sobre desarrollo rural endógeno y participación ciudadana; analizar las prácticas de participación ciudadana en los procesos de desarrollo rural endógeno en el cantón Limón Indanza en el periodo 2009-2019; estructurar componentes de una metodología que incorpore la participación ciudadana en el desarrollo rural endógeno para el caso cantón Limón Indanza; y, validar la metodología de participación ciudadana mediante criterio de expertos. Como aspecto adicional, se realiza un análisis basado en los métodos multivariados para la caracterización del desarrollo rural sostenible en el Cantón Limón de la provincia de Morona Santiago, Ecuador.

Antes de abordar el desarrollo del trabajo de investigación, es necesario realizar una contextualización de la situación que se desea evaluar, dado que, como se mencionó anteriormente, los diferentes niveles de planificación deben estar interconectados.

En el contexto ecuatoriano, en febrero de 1997 el movimiento de cientos de miles de ecuatorianos que se manifestaron en las calles, ejerciendo en la práctica el derecho de revocatoria de mandato se unieron en asambleas locales para luchar contra el gobierno y se crearon espacios de concertación con nuevas formas de ejercicio de la democracia, lo que 
colocó en primer plano de la vida nacional el tema de la participación ciudadana y abrió un tercer momento (Hidalgo et al., 1999).

En la Constitución de 2008 la participación es un elemento importante, pues se reconoce la presencia de múltiples actores de la sociedad: mujeres, indígenas, jóvenes, niños, discapacitados, consumidores; y se establecen nuevas y diversas formas de participación: fiscalización, consulta, planificación y revocatoria, en múltiples ámbitos y temáticas educación, salud, medio ambiente, políticas de la mujer e infancia, etc.

Tanto la Constitución como un conjunto de leyes ecuatorianas norman la relación entre participación ciudadana y desarrollo local; con base en ello, se destaca que la participación ciudadana se consagra en el artículo 95 de la Constitución de la República del Ecuador, indicando que las personas y organizaciones participarán activamente y de manera protagónica en la construcción del poder ciudadano, rigiéndose por una serie de principios de igualdad. Asimismo, en el artículo 96, se reconocen diversas formas de organización en la sociedad para la participación (ANC, Constitución de la República del Ecuador, 2008).

La Ley Orgánica de Participación Ciudadana (Asamblea Nacional, Ley Orgánica de Participación Ciudadana, 2010) establece en su Artículo 1 que su objeto es fomentar y garantizar que los ciudadanos y las ciudadanas ejerzan su derecho a la participación ciudadana, con la representación de diversas comunidades, grupos étnicos y organizaciones establecidas de manera lícita, promoviendo de esta manera la democracia participativa; por otra parte, en el artículo 29, se define al Poder Ciudadano como “...el resultado del proceso de la participación individual y colectiva de las ciudadanas y ciudadanos de una comunidad..." (p. 17).

Finalmente, el Código Orgánico de Organización Territorial Autonomía y Descentralización (Asamblea Nacional del Ecuador, 2010) indica en su artículo 302, que "El derecho a la participación ciudadana se ejercerá en todos los niveles de los gobiernos autónomos descentralizados a través de los mecanismos de la democracia representativa, directa y comunitaria" (p. 120). Esto le da fortaleza al engranaje nacional para el desarrollo desde las comunidades o lo local; a partir de esto, se ha creado una estructura basada en el Gobierno Autónomo Descentralizado (GAD), responsable de guiar y coordinar el Plan de Desarrollo y Ordenamiento Territorial (PDOT); lo cual existe a nivel de parroquias, cantones y provincias.

Comparando lo que dicen Alburquerque y Cortés (2001) con la situación del país, se puede observar que al analizar diferentes PDOT cantonales, en su mayoría, se evidencia la falta de participación ciudadana y, en algunos casos, aunque esta existe, no se encuentra dentro del nivel necesario para garantizar la excelencia del proceso de planificación pese a la existencia de un marco legal y una Ley Orgánica de Planificación dentro del Estado ecuatoriano.

Entonces se podría decir que, por la falta de participación ciudadana, la identificación de los recursos endógenos se ve ampliamente limitada, pues en algunos casos, en los cantones se 
lleva a cabo el diseño de los PDOT, solo desde un enfoque técnico y no se da la importancia necesaria al verdadero objetivo de la planificación territorial local, los habitantes, lo que se produce y lo que en conjunto se quiere lograr, requiriendo de verdadera participación.

En el estudio, el análisis se centró en la participación ciudadana del cantón Limón Indanza, en el marco de las posibles potencialidades endógenas que posteriormente se pudieran impulsar. Con base en una revisión del PDOT-2015 se pudo determinar que este cantón se encuentra ubicado en la zona sur de la provincia Morona Santiago, con una extensión territorial de $1.821 \mathrm{~km}^{2}$ y consta de 6 parroquias, de las cuales una (1) es urbana y cinco (5) son rurales, listadas a continuación:

- Parroquia urbana: General Leónidas Plaza Gutiérrez.

- Parroquias rurales: Yunganza, Santa Susana de Chiviaza, Indanza, San Antonio y San Miguel de Conchay.

Al momento de la elaboración del PDOT, Limón Indanza tenía una población de 9.722 habitantes, integradas en 70 comunidades; la población urbana es de 3.523 habitantes representando el 36,23\% del total cantonal, mientras que la población rural es de 6.199 habitantes y corresponde al 63,77\% (P.D.O.T de Limón Indanza, 2014); según la proyección de población para 2020 la población total del cantón alcanzaría 10.349 habitantes y se estima que el 65,7\% son personas de 18 años o más, es decir, unos 5.900 habitantes (Instituto Nacional de Estadística y Censos (INEC), 2020).

En términos económicos, la zona rural cuenta con un total de 2.353 ocupados en el sector primario, mientras que en la zona urbana este sector tiene ocupadas, apenas, a dos (2) personas; entonces, se podría afirmar que en la zona rural se encuentra un gran potencial para la producción de recursos endógenos. Por otra parte, en el PDOT se identificaron variables biofísicas que sirvieron para determinar problemas y potencialidades; allí se observa el mapeo de actores sociales, la identificación de potencialidades y problemas como la falta de motivación de los ciudadanos para participar.

En este sentido, la problemática principal identificada es la escasa participación ciudadana en los asuntos de planificación local y seguimiento de los proyectos de desarrollo, posiblemente relacionado con poca motivación o falta de incentivos sociales para discutir acerca de los problemas comunes y sobre las perspectivas de desarrollo local.

Es importante señalar que, uno de los objetivos en el Plan Nacional de Desarrollo 2017-2021 es incentivar una sociedad participativa, con un Estado cercano al servicio de la ciudadanía para lograr potenciar a cada sector, especialmente desde el cantón (Consejo Nacional de Planificación (CNP), 2017). Esto debería promover la participación, tanto desde las bases comunitarias como desde las instituciones gubernamentales, sin embargo, se observa pasividad en gran parte de las comunidades de Limón Indanza. Resulta claro que el cumplimiento del mandato constitucional acerca de la construcción de una democracia 
participativa se encuentra afectado y reducido si las personas y comunidades no se organizan, reúnen para discutir y realizan el seguimiento de lo establecido en los PDOT.

El desarrollo local es un proceso dinámico de constantes cambios cuyo objetivo principal es mejorar el bienestar, calidad de vida y autorrealización del sector rural, en referencia a su situación económica y social considerando la desigualdad y las condiciones culturales de las comunidades (Guinjoan et al., 2016).

Los enfoques del desarrollo rural endógeno hacen hincapié en el máximo aprovechamiento de los recursos locales, incluyendo el capital humano, para incorporar a los actores locales (grupos de interés económico y social, población en general e instituciones públicas y privadas representativas), en la participación de la toma de decisiones sobre las estrategias para la realización de los proyectos seleccionados, el proceso de implementación y la selección de las distintas medidas a seguir, convirtiendo así a la participación ciudadana en un medio para el desarrollo rural.

Los factores determinantes del desarrollo económico local se ubican en: demografía, estructura económica, ingresos, servicios básicos, ubicación espacial y gobernanza; en tanto que se deben considerar otros, tales como: ubicación, factores físicos, infraestructura, recursos humanos, capital y finanzas, conocimiento y tecnología, estructura industrial, calidad de vida, cultura empresarial, identidad e imagen comunitaria, y capacidad institucional (Pavel \& Moldovan, 2019).

La participación ciudadana es un concepto altamente polisémico y, a su vez, complejo, que contribuye a cambios sociales significativos y que requiere de un costo social importante. Según Bednarska-Olejniczak et al. (2020), los conceptos teóricos de la participación ciudadana en las actividades de las instituciones públicas, como brazo ejecutor de las políticas gubernamentales que rigen la actuación y el destino del Estado

Finalmente, es necesario mencionar el modelo de Sherry Arnstein, conocido como la Escalera de Arnstein, presentado por esta autora en 1969, el cual constituye una de las principales teorías de la participación ciudadana; distingue entre ocho tipos de participación, ordenado jerárquicamente de acuerdo con el aumento del poder de decisión de los interesados, (Bednarska-Olejniczak et al., 2020). A continuación, se muestra una representación del esquema de dicha teoría: 
Figura 1. Escalera del modelo de participación ciudadana de Arnstein.

\begin{tabular}{|c|c|c|}
\hline Nivel & Tipo de participación & $\begin{array}{l}\text { Influencia de la ciudadanía } \\
\text { en la decisión final }\end{array}$ \\
\hline 8 & Control ciudadano & \multirow{3}{*}{ Grados de poder ciudadano } \\
\hline 7 & Poder delegado & \\
\hline 6 & Asociación & \\
\hline 5 & Apaciguamiento & \multirow{3}{*}{ Grados de simbolismo } \\
\hline 4 & Consulta & \\
\hline 3 & Información & \\
\hline 2 & Terapia & \multirow{2}{*}{ No participación } \\
\hline 1 & Manipulación & \\
\hline
\end{tabular}

Elaborado por: El autor. Fuente: Bednarska-Olejniczak et al. (2020)

Cada uno de estos niveles hace referencia a la capacidad de decisión de los ciudadanos y de la influencia de la administración gubernamental sobre los mismos (Bednarska-Olejniczak et al., 2020).

Bajo este contexto, la investigación tuvo como objetivo plantear una metodología que contribuya a mejorar la participación ciudadana, al mismo tiempo que se buscó dilucidar cuáles son las características de los niveles de participación, mediante el contraste de las percepciones de los responsables institucionales del cantón Limón Indanza y de los ciudadanos. Se consideró importante el proceso de planificación que se ha llevado en el cantón Limón Indanza, desde el planteamiento del PDOT hasta la forma en que los habitantes han participado en su seguimiento.

\section{Metodología}

La investigación es de tipo mixto, ya que se aplicaron los métodos cuantitativo y cualitativo. El primero, se define mediante un enfoque no experimental-longitudinal, para medir el nivel de acogida que tiene la participación ciudadana mediante el análisis y procesamiento de la información estadística obtenida en la encuesta, la que se realizó sobre la población de 18 años o más, residentes del cantón y cuyo tamaño de la muestra se calculó según:

$$
n=\frac{N \cdot Z_{\alpha / 2}^{2} \cdot p \cdot q}{e^{2} \cdot(N-1)+Z_{\alpha / 2}^{2} \cdot p \cdot q}
$$




$$
n=\frac{5.900 \cdot 1,96_{\frac{\alpha}{2}}^{2} \cdot 0,5 \cdot 0,5}{0,07^{2} \cdot(5.900-1)+1,96_{\frac{\alpha}{2}}^{2} \cdot 0,5 \cdot 0,5}
$$

Siendo el tamaño de muestra final de $\mathrm{n}=190$ personas de 18 años o más.

El segundo enfoque, se basó en el análisis de la información generada en discusiones de grupos focales y entrevistas, los cuales se centraron en la indagación de opiniones o posturas de dirigentes de varias instituciones, integrantes de la comunidad y líderes comunitarios, así como a ciudadanos que estuvieron presentes en la elaboración del Plan de Desarrollo y Ordenamiento Territorial (PDOT); esto permitió obtener la percepción de los habitantes del cantón respecto a la participación ciudadana. Asimismo, se utilizaron los métodos analítico y sintético. Asimismo, la investigación tiene un alcance analítico y propositivo; mediante la comparación se analizó la percepción de la participación ciudadana desde los puntos de vista institucional y ciudadano.

La metodología propuesta se basó en la participación ciudadana mediante el modelo LUDO, el cual permite involucrar a los habitantes y gobernantes en un ejercicio de evaluación de la situación actual y situación deseada para la localidad, la definición del marco estratégico y de la cantidad de poder que se transferirá a los ciudadanos, el diseño de los proyectos con objetivos claros y medibles, así como la distribución de responsabilidades y establecimiento de las acciones a seguir de manera conjunta. Finalmente, esta propuesta fue evaluada por expertos en planificación territorial mediante el uso del Método Delphi.

\section{Resultados}

Después de la aplicación de los diferentes instrumentos de recolección de datos, se presentan los siguientes resultados:

La encuesta se realizó en un total de 190 habitantes del cantón Limón Indanza y se analizó su confiabilidad mediante el estadístico Alfa de Cronbach, cuyo resultado fue aceptable según el valor de 0,706. Como se puede apreciar en la figura 1, el 50,7\% de los participantes de la encuesta son mujeres, un 26,2\% del total tienen edades entre 45 y 54 años. En general, el 73,9\% tienen disposición de participar en sesiones parroquiales y cantonales, 92,6\% tienen intereses en los asuntos públicos y 15,3\% están dispuestos a acudir a las sesiones del consejo cantonal. 
Figura 2. Principales resultados de la encuesta a 190 residentes del Cantón Limón Indanza.
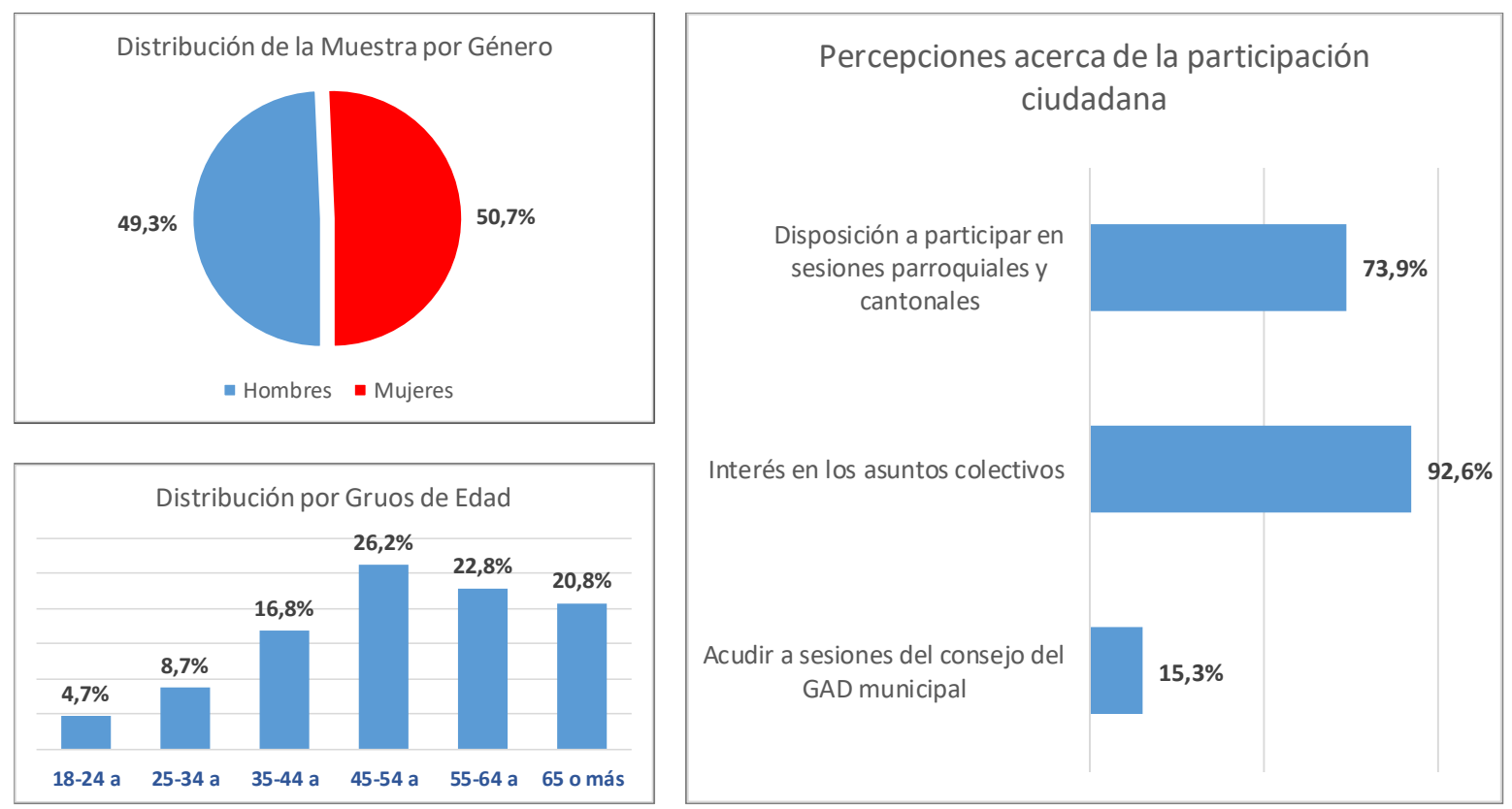

Elaborado por: El autor.

Se entrevistó a un grupo de funcionarios del cantón Limón Indanza, quienes afirman que, respecto al ejercicio de la participación ciudadana y la actuación de los funcionarios se tiene que el control del seguimiento de los proyectos recae en el criterio propio de cada funcionario responsable, por lo que se percibe heterogeneidad dentro de la organización del gobierno local. Por otra parte, se emplean estrategias como el Gobierno Abierto, Innovación abierta con compromiso y Gobernanza Pública Inteligente, con el fin de establecer contacto con los ciudadanos; en este sentido, el personal del gobierno local promueve la participación ciudadana mediante convocatorias a los actores sociales, rendición de cuentas y publicación en los medios digitales. Sin embargo, los funcionarios no manejan el término "Contraloría Social", lo que dificulta el cumplimiento de la participación ciudadana, a pesar de que el proceso de rendición de cuentas se hace a través de las consultas ciudadanas. Los empleados municipales perciben que el área productiva (agro), educación, salud y vialidad son las áreas que requieren mayor atención para el desarrollo del cantón; también, consideran que es necesario fortalecer la formación y capacitación en emprendimiento e innovación, economía popular, desarrollo agropecuario, turismo y desarrollo sustentable.

Asimismo, a partir de las entrevistas a los residentes del cantón se conoció que se realizan convocatorias desde el gobierno local, pero no son consecuentes; esto produce pérdida de la dinámica del seguimiento de los proyectos y de la participación ciudadana. Asimismo, se tiene que no se actualizan las necesidades del cantón dentro de la planificación local; no existe un esquema claro y formal de la participación ciudadana dentro del cantón y es 
necesaria la socialización de los logros y problemas presentados en la ejecución de los proyectos.

Finalmente, se realizaron dos sesiones de grupo focal, en los cuales se expresó que los proyectos del cantón son elaborados en oficina por un grupo de personas determinadas, por lo que no se convoca a las representaciones comunitarias; pero se considera que las mesas de diálogo son el principal instrumento de participación de las comunidades. No existe una actualización del PDOT o su respectiva publicación, en tanto que tampoco existe claridad en la información desde el gobierno municipal. Asimismo, la participación de las comunidades del cantón en la discusión de los problemas y evaluación de los proyectos no es equilibrada, ya que hay regiones que participan y otras que no lo hacen por falta de convocatoria o de información. En cuanto a los niveles de comunicación, se observa que el gobierno local publica información limitada en las Redes Sociales como Facebook, pero no cumple con la formalidad ante las comunidades.

\section{Metodología propuesta para la participación ciudadana}

Al observar las falencias encontradas en el Cantón Limón Indanza respecto a la participación ciudadana, se propone que el mismo ejecute una estrategia basada en el modelo LUDO de gobierno abierto. En este se destaca la importancia del liderazgo institucional como promotor de la participación ciudadana, dado que los recursos financieros se encuentran de este lado de la balanza, por lo tanto, el poder, el cual es necesario distribuir mediante mecanismos de participación y distribución del ingreso a través de proyectos que beneficien a la comunidad y la involucren permanentemente en el desarrollo de la localidad.

Como premisa esencial para la participación ciudadana se establecen tres principios que direccionarán el ejercicio de la misma, los cuales son: transparencia, participación y colaboración. A continuación, se presentan los lineamientos básicos en el marco de estos principios:

Figura 3. Principios del modelo LUDO en el diseño de la metodología de participación ciudadana.

\begin{tabular}{|c|c|c|}
\hline \multicolumn{3}{|c|}{ Modelo LUDO de gobierno abierto } \\
\hline & iativas institucion & \\
\hline Transparencia & Participación & Colaboración \\
\hline $\begin{array}{l}\text { Publicación de } \\
\text { documentos. } \\
\text { - Rendición de } \\
\text { cuentas. } \\
\text { - Contrataciones } \\
\text { abiertas. }\end{array}$ & $\begin{array}{l}\text { - Convocatorias } \\
\text { periódicas. } \\
\text { - Abarcar toda la } \\
\text { extensión del } \\
\text { cantón } \\
\text { (comunidades). }\end{array}$ & $\begin{array}{l}\text { - Participación } \\
\text { institucional y de } \\
\text { las comunidades } \\
\text { en la resolución } \\
\text { de problemas y } \\
\text { en la } \\
\text { planificación } \\
\text { local. }\end{array}$ \\
\hline
\end{tabular}

Fuente: Elaboración propia. 
Con base en el Modelo LUDO de participación, se propone la siguiente metodología para la participación ciudadana en la planificación local del Cantón Limón Indanza:

1. Convocar a los representantes comunitarios a la discusión de proyectos de desarrollo propuestos institucionalmente mediante mesas de diálogo, donde participen el Gobierno Local y los ciudadanos.

2. Actualizar el listado de organizaciones, grupos sociales y actores del cantón Limón Indanza, con el fin de cubrir integralmente la participación en todos sus ámbitos.

3. Recoger diferentes propuestas de proyectos de desarrollo desde las comunidades, procurando la participación de todas las comunidades y estableciendo mecanismos de participación flexibles.

4. Crear una agenda de discusión y valoración de los proyectos, con base en la búsqueda de información confiable que permita establecer marcos de referencia, realizar cálculos y obtener informes confiables para la toma de decisión.

5. Contratar asesoría técnica cualificada para el diseño de los proyectos y la evaluación de las potencialidades y viabilidad de los mismos.

6. Priorizar los proyectos que tengan carácter vital para el crecimiento y desarrollo del cantón.

7. Diseñar un método de referéndum para seleccionar proyectos.

8. Permitir la verificación de los avances de la ejecución de proyectos, así como la contraloría social facilitada con la publicación de las cuentas en la prensa local y mediante los medios digitales.

9. El gobierno local debe dirigir también la atención a proyectos de menor escala en cada comunidad, con el objeto de integrar a la comunidad en todos los niveles posibles.

10. Establecer una agenda de revisión con los representantes y comunidades.

11. Diseñar y establecer las políticas públicas mediante consulta con las comunidades.

12. Ofrecer, desde la institucionalidad, a los ciudadanos la posibilidad de capacitarse en temas de planificación y desarrollo local; esto servirá de apoyo para el aumento de la calidad de las propuestas y el desarrollo de los proyectos, con base técnica, desde las bases de la comunidad. También, contribuirá notablemente en la evaluación de los proyectos en marcha. 
13. Construir un sistema de información de la gestión de proyectos, con lo cual se puede hacer el monitoreo y compartir la información.

14. La socialización de los resultados, del estado de los proyectos y del presupuesto es fundamental para establecer la transparencia institucional, así como también para crear mayor nivel de pertenencia y preocupación de los ciudadanos por los asuntos públicos que afectan a su comunidad y nivel de vida; por ello, es necesario que se establezca la periodicidad de publicación y presentación de información a la comunidad, mantener la disponibilidad de los datos y el respectivo acceso a los mismos.

Este modelo es pertinente para la planificación del cantón Limón Indanza porque se encuentra en armonía con lo establecido en la Constitución, a la vez que se fundamenta en el paradigma de "gobierno abierto" y en los valores de transparencia, participación y colaboración.

\section{Discusión}

Según los hallazgos de la investigación, el Cantón Limón Indanza cuenta con las potencialidades para ubicarse dentro de un proceso de desarrollo creciente; no obstante, se evidencia escasa participación ciudadana en el diseño de los planes y proyectos de desarrollo, así como en el seguimiento de los proyectos actuales, lo que dificulta el desarrollo endógeno a partir de la relación directa entre los habitantes y el presupuesto, quedando, por lo tanto, a discreción de las autoridades institucionales la toma de decisiones. Al respecto, según Steel y Mitchell (2017), el desarrollo endógeno se encuentra en el extremo opuesto del continuo de desarrollo, proporcionando el mayor grado de hegemonía local.

Según la encuesta aplicada, el 73,9\% tienen disposición de participar en sesiones parroquiales y cantonales, mientras que el 92,6\% tienen intereses en los asuntos públicos, pero 15,3\% están dispuestos a acudir a las sesiones del consejo cantonal. Estos resultados reflejan el interés de los habitantes del cantón en los asuntos que involucran el desarrollo local, sin embargo, una proporción muy baja están dispuestos a participar directamente; esto puede estar asociado con la falta de acceso a la información, falta de convocatoria, escasa relación entre las autoridades y la población, percepción respecto a la poca importancia que se le da a la participación.

Asimismo, se evidencia que no existe una metodología de participación ciudadana, por lo que carece de los instrumentos necesarios para el empoderamiento de los locales en cuanto al destino de los recursos y el uso de las potencialidades. Mubita, Libati y Mulonda (2017), refieren que los críticos de la participación ciudadana afirman que esta no conduce al empoderamiento de los locales, porque las metodologías participativas no logran cambiar y desafiar las estructuras burocráticas, centralizadas y administrativas que controlan la toma de decisiones y la asignación de recursos. 
De acuerdo con Sáenz Pinzón (2017), es necesario plantear metodologías y mecanismos de participación ciudadana que acompañen los esfuerzos institucionales del desarrollo local, como base del fortalecimiento de una democracia participativa. En este sentido, superar las barreras de la participación será un requisito esencial para el empoderamiento de la ciudadanía. Como lo mencionan Sandoval et al.(2015), barreras como la ubicación, distancia, poca disponibilidad de tiempo de las personas ocupadas y el lenguaje técnico, ponen en riesgo la capacidad de participación de los ciudadanos en las actividades de planificación y seguimiento de los proyectos locales; asimismo, son desventajas que pueden marcar la diferencia entre diferentes grupos de participantes, por lo que la participación ciudadana observada por las instituciones estará sesgada por la falta de representación de determinados grupos.

La metodología propuesta fue evaluada por un grupo de expertos profesionales, quienes validaron la misma mediante el método Delphi; en general, los expertos indicaron que la metodología se ajusta al diseño LUDO de participación ciudadana. De acuerdo con Camacaro Sierra et al. (2012), el uso del método Delphi es de gran utilidad para validaciones, dado que el aporte de la experiencia y los conocimientos de los expertos, desde el punto de vista individual y grupal, respaldan o refutan los planteamientos que se evalúan.

La metodología planteada establece responsabilidades de manera bidireccional; por lo que, tanto los actores sociales como los representantes institucionales tienen el deber de cumplir con los compromisos establecidos, lo que representa un enfoque político, dentro de un marco de solidaridad, cooperación y búsqueda del beneficio común para el desarrollo del cantón. Según Mubita et al. (2017), la participación debe ser considerada como política ya que está condicionada por el marco institucional y los antecedentes políticos de los participantes.

Es necesario que las metodologías de participación se adapten a las cualidades propias de cada región o localidad, de modo que es claro comprender que lo que funciona en un lugar no necesariamente funcionará en otro, ya que las condiciones cambian. En correspondencia con esto, García-Montes y Arnanz Monreal (2019) indican que, el desarrollo de metodologías de participación ciudadana para el desarrollo local debe favorecer la creatividad de las comunidades, la liberación, la construcción de redes y la transformación del entramado social, mediante el crecimiento colectivo.

\section{Conclusiones}

- De acuerdo con los resultados obtenidos se concluye que, en Ecuador, la participación ciudadana es un mecanismo político consagrado en la Constitución de 2008, que permite a las comunidades realizar aportes en la planificación local, así como también realizar el control social sobre el uso de los fondos públicos en proyectos de desarrollo. 
- Se nota cierta dispersión en las convocatorias a participación desde la organización cantonal. Es necesario formalizar los mecanismos y esquemas de participación ciudadana en el cantón Limón Indanza, con el fin de obtener un mayor nivel de participación y compromisos bidireccionalmente.

- Asimismo, se plantean recomendaciones dirigidas a mejorar los niveles de participación ciudadana en la planificación local, tales como: incorporar a las comunidades en la discusión de los planes y proyectos del cantón; actualizar el PDOT, mediante la incorporación de diferentes actores sociales del cantón y representación de todas las comunidades y grupos; priorizar la ejecución de proyectos de desarrollo rural, acompañado de la construcción de vialidad que impulse al desarrollo; y, estructurar mecanismos de participación ciudadana permanente y formalizarlo, al mismo tiempo que se unifican los criterios intra institucionales para mejorar la cooperación de los funcionarios.

\section{Referencias bibliográficas.}

Alburquerque, F., \& Cortés, P. (2001). Desarrollo económico local y descentralización en América Latina. Naciones Unidas, CEPAL.

Constitución de la República del Ecuador, $449 \quad 129 \quad$ (2008). https://www.asambleanacional.gob.ec/sites/default/files/private/asambleanacional/fi lesasambleanacionalnameuid-29/constitucion-republica-inc-sent-cc.pdf

Ley Orgánica de Participación Ciudadana, Pub. L. No. Registro Oficial Suplemento 175, 25 (2010).

Bednarska-Olejniczak, D., Olejniczak, J., \& Svobodová, L. (2020). How a Participatory Budget Can Support Sustainable Rural Development-Lessons From Poland. Sustainability, 12(7), 1-29. https://doi.org/10.3390/su12072620

Camacaro Sierra, L., Rodríguez Silva, M., Caldera de Ugarte, N., \& Cestary Colmenares, J. (2012). Visión actual del desarrollo turístico urbano de Maracaibo. Método Delphi. Revista de Ciencias Sociales (Ve), XVIII(3), 430-448. https://www.redalyc.org/pdf/280/28024392004.pdf

Consejo Nacional de Planificación (CNP). (2017). Plan Nacional de Desarrollo 2017-2021Toda una Vida (Resolución N. ${ }^{\circ}$ CNP-003-2017; p. 148). Secretaría Nacional de Planificación y Desarrollo, Senplades. https://www.planificacion.gob.ec/wpcontent/uploads/downloads/2017/10/PNBV-26-OCT-FINAL_0K.compressed1.pdf

García-Montes, N., \& Arnanz Monreal, L. (2019). Metodologías participativas para la planificación de la sostenibilidad ambiental local. El caso de la Agenda 21. EMPIRIA. 
ISSN: 2600-5859

www.concienciadigital.org

Vol. 4, N¹.1, p. 67-82, Febrero, 2021

Revista de Metodología de Ciencias Sociales, 43, 109-133. https://doi.org/10.5944/empiria.43.2019.25354

Guinjoan, E., Badia, A., \& Tulla, A. F. (2016). El nuevo paradigma de desarrollo rural. Reflexión teórica y reconceptualización a partir de la «Rural Web». Boletín de La Asociación de Geógrafos Españoles, 71, 179-204. https://doi.org/10.21138/bage.2279

Hidalgo, M., Guerrero, F., Larrea, A. M., Larrea, F., Muñoz, J. P., Ortiz, S., Tola, B., Torres D., V. H., \& Unda, M. (1999). Ciudadanías emergentes: Experiencias democráticas de desarrollo local (1era ed.). Abya-Yala.

Instituto Nacional de Estadística y Censos (INEC). (2020). Proyecciones Poblacionales [Institucional - Oficial]. https://www.ecuadorencifras.gob.ec/proyeccionespoblacionales/

Mubita, A., Libati, M., \& Mulonda, M. (2017). The Importance and Limitations of Participation in Development Projects and Programmes. European Scientific Journal, 13(5), 238-251. https://doi.org/10.19044/esj.2017.v13n5p238

Pavel, A., \& Moldovan, O. (2019). Determining Local Economic Development in the Rural Areas of Romania. Exploring the Role of Exogenous Factors. Sustainability, 11(282), 1-24. https://doi.org/10.3390/su11010282

Sáenz Pinzón, G. P. (2017). Bosquejo de una propuesta de presupuesto participativo para los municipios de Colombia. Administración y Desarrollo, 47(2), 110-135.

Sandoval, C., Sanhueza, A., \& Williner, A. (2015). La planificación participativa para lograr un cambio estructural con igualdad (Manuales de la CEPAL ISSN 2518-3923; p. 74).

CEPAL. https://repositorio.cepal.org/bitstream/handle/11362/39055/7/S1501278_es.pdf

SENPLADES. (2012). Guía metodológica de planificación institucional (p. 48). Secretaría Nacional de Planificación y Desarrollo. https://www.planificacion.gob.ec/wpcontent/uploads/downloads/2016/03/GUIA-DE-PLANIFICACIONINSTITUCIONAL.pdf

Steel, C. E., \& Mitchell, C. J. A. (2017). Economic Transition in the Canadian North: Is Migrant-Induced, NeoEndogenous Development Playing a Role? Journal of Rural and Community Development, 12(1), 55-74.

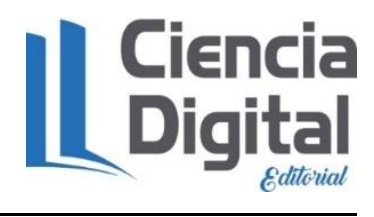




\section{PARA CITAR EL ARTÍCULO INDEXADO.}

Argudo Domínguez, B. A., \& Castillo Ortega, Y. (2021). Metodología de participación ciudadana en el proceso de desarrollo rural endógeno en el caso Cantón Limón Indanza . ConcienciaDigital, 4(1.1), 67-82. https://doi.org/10.33262/concienciadigital.v4i1.1.1547

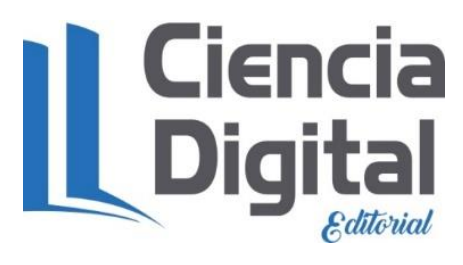

El artículo que se publica es de exclusiva responsabilidad de los autores y no necesariamente reflejan el pensamiento de la Revista Conciencia Digital.

El artículo queda en propiedad de la revista y, por tanto, su publicación parcial y/o total en otro medio tiene que ser autorizado por el director de la Revista Conciencia Digital.

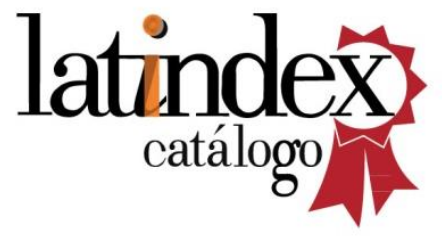

